\title{
Field Investigations: An Overlooked Form of Laboratory Experience
}

\section{Prof. David F. Radcliffe, Swinburne University of Technology}

Dr. Radcliffe's research focuses on the nature of engineering; engineering habits of mind, how engineering knowledge is created and shared and how it is learned especially outside the classroom. Over the past 30 years, he has conducted field research on the practice of engineering design, new product development and innovation in variety of industries, in large and small firms with an emphasis on design thinking, most recently in relation to sustainability. He also studies engineering education as a complex system, and the design and evaluation of next generation learning environments. This research is intrinsically multidisciplinary and draws on methodologies from the humanities, social and behavioral sciences and involves collaboration with anthropologists, learning scientists, librarians, designers, and architects.

\section{Dr. Mary K. Pilotte, Purdue University, West Lafayette}

Mary Pilotte is Associate Professor of Engineering Practice in the School of Engineering Education at Purdue University, West Lafayette, Indiana. She is an instructor for Multidisciplinary engineering coursework, and is Director of the undergraduate Interdisciplinary Engineering Studies and Multidisciplinary Engineering program. She also is an instructor in executive and global MBA programs. With over 20 years of industrial work experience, and supportive of her academic roles, Mary actively leads academic outreach to industrial firms to develop in/out of classroom, project-based, active learning through identification of authentic, in-context problem scenarios and the embedded cadence of practice. Pilotte's research interests involve understanding generation-based engineering culture, identity, and communication in the context of professional engineering practice. Expanded interests include understanding student benefits associated with in-context active learning, and the intersection of engineering education and neurodiversity. She holds a Bachelor of Science degree in Organizational Leadership and Supervision from Purdue University, an MBA from the Goizueta School of Business, Emory University, Atlanta, Georgia, and a Ph.D. in Engineering Education from Purdue University. 


\title{
Field Investigations: An Overlooked Form of Laboratory Experience
}

\begin{abstract}
The types of learning activities that are accepted as constituting a legitimate laboratory experience have expanded considerably over the past decade or so. Traditionally, laboratory instruction was taken to involve hands-on activities around measuring quantitative, technical data associated with some physical phenomenon or the performance of an engineered artefact. More recently virtual laboratories based on computer simulation and remote labs where physical or virtual equipment is accessed at a distance using the internet have become accepted under the umbrella of "laboratory". The proposed new ABET Criteria 3 (Student Outcomes) has broadened somewhat in respect of experimentation, thereby presenting an opportunity for discussion around laboratory contexts.
\end{abstract}

This paper argues we should adopt a more expansive understanding of what counts as a "laboratory"; one that recognizes the reality that practicing engineers must be adept at creating and conducting investigations that take into account not only technical factors but also the sociocultural, economic and even ethical aspects and impacts of an engineering project. This might include gathering data of many types; technical and non-technical, qualitative and quantitative, captured and analyzed in a variety of media. Interpreting, making sense of, and development of inferences from such diverse data sets is a developmental extension of the skills acquired by a student in a traditional, technical laboratory setting. As such, what might be termed "sociotechnical" laboratory experiences add to, rather than replace, laboratory instruction as we have known it. This paper focuses on one ubiquitous form of engineering experimentation with sociotechnical dimensions, i.e. fieldwork. It uses a case study based on a novel Study Abroad program to illustrate the design and implementation of a socio-technical laboratory, one that emulates an experience of global engineering fieldwork.

\section{Introduction}

The practice of engineering is a profession that engages the material world. The work engineers undertake requires being in and around the processes, artefacts, and users of the solutions they develop. This necessarily leads to being involved in investigations "in the field" where a wide range of technical and non-technical data is gathered or generated, and situational awareness is central to their analysis and recommendations based upon the conclusions drawn in the given situation. During their career, most engineers have undoubtedly faced the urgent matter of being sent to an installation site or to visit a supplier to investigate a technical or production problem. The location of the stakeholders and/or project site could require travelling out of state, or even out of their country. Yet, they will urgently seek to trouble shoot a problem they may have never seen, with a local team they probably have never met, in a location and circumstance that may have limited access to the tools and resources normally available to them.

In this recognizable, yet unfamiliar situation, the visiting engineer must utilize local resources to identify and gather what they perceive to be relevant data without the benefit of home-base equipment. Quickly, through this initial investigation, a better definition of the problem hopefully emerges. Preliminary findings lead the engineer to report back to their team, and make 
recommendations for next steps toward long-term problem resolution. Success in such prototypical field investigations requires engineers make adjustments to work cadence and cultural practices. This involves meeting and interacting with the local team socially after what they consider normal work hours. This engineering experience highlights how the technical orientation toward problem solving is inextricable from the socio-cultural contexts they are situated within [1].

Conducting this form of field investigation draws upon a wide variety of knowledge, skills and abilities, the foundations of which are laid at the university, as well as in earlier formal education and broader life experiences. Learning to do this work is not something that can be reduced to a simple step-wise procedure or set out in a formulated process. It involves understanding technical information within the unique operating context in which the technical reality exists. Following on, one must be able to interpret the information, communicating it to others through their particular lens, be it business, marketing, operations, finance, corporate HQ or community impact. Whether the fieldwork takes place across the country or on the other side of the world, is informed by an appreciation of the background, history and maybe the geography of the place in question, the prevailing politics and power relationships and the underlying social and cultural mores. While engineering programs are becoming more intentional about developing some of the foundational knowledge, skills and abilities that underpin successful fieldwork, typically these are fostered separately in disparate parts of the curriculum. For example, cultural awareness is usually part of study abroad preparation, while fostering empathy as part of a user-centered approach to design is commonly associated with service learning programs. Likewise, an understanding of people and place is scattered across various liberal arts courses. It is unusual for any of these approaches and concerns to be associated with laboratory instruction.

This paper makes the case that in preparing graduates for future engineering practice we need to broaden our conception of what constitutes as a "laboratory" experience to include open-ended investigations set in unfamiliar socio-material contexts. We envisage a variety of "lab" types strategically located across the curriculum, each designed to develop specific abilities linked to conducting authentic engineering investigations. The nature and timing of each type of lab experience would be positioned to be developmentally appropriate, becoming increasingly more contextual and moving beyond purely technical investigations. More socio-technical type "laboratories" would likely occur in the later years of the degree program. This paper opens by briefly exploring the history and evolution of the laboratory in engineering programs over the past century and then critically reviews the desired graduate outcomes that should result from students engaging in experiments and other forms of investigation. It concludes with a case study of one instance of a novel study abroad course, focused on providing engineering students with a kindred fieldwork experience.

Evolution of Laboratory Instruction in Engineering Education

Laboratory is a defining facet of engineering education; an integral part of the student experience. However, the nature of what constitutes a "laboratory" has changed dramatically since engineering schools were founded in the mid- $19^{\text {th }}$ century. Initially laboratories involved investigating the operation of actual engineering artefacts or equipment (for example a working locomotive used to measure thermodynamic performance) or conducting survey work outdoors. Remnants of this contextual and artefact-based approach are still found in some electric machine 
laboratories, in mechanical dissection labs and in some agricultural engineering programs. Over time, the laboratory experience evolved to be more associated with conducting predetermined studies to demonstrate scientific phenomena (e.g. physics and chemistry labs), illustrate concepts in the engineering sciences (e.g. materials properties, basic mechanics, thermo-fluids) or working with elemental technology componentry (e.g. circuits lab). The assumption has been that these experiences were hands-on, where the students engaged with the practical side of engineering to complement the theory provided in lectures. Yet the nature of the laboratory has been changing rapidly and the learning outcomes arising from labs are not as clearly defined as perhaps we once assumed [2].

The earliest review of engineering education, conducted just one a century ago, made the following observation about the centrality of the laboratory in instruction:

"Since the engineering schools entered upon their remarkable development fifty years ago the conditions of industrial production have changed, new fields of engineering have been developed, the professional ideals of the engineer have grown more definite, laboratory work has won recognition as an essential element of all instruction in science, and educational theory and practice have been brought within the range of scientific test [3]."

Feisel and Rosa [4] published a wide-ranging review of the history of instructional laboratories in engineering schools, observing that there has been a tension between the relative importance of "theory" and "practice" since formal, college-based engineering education programs began in the US. Initially the prime focus was on the practical aspect of engineering. Over subsequent decades it would seem that the ubiquity and centrality of the laboratory as an indispensable part of engineering instruction was taken for granted. For instance, Feisel and Rosa [4] note that the first accreditation criteria published in 1933 by the Engineering Council for Professional Development (precursor of ABET) did not explicitly refer to laboratories. They also state that:

"Engineering programs required science and mathematics, but drafting and laboratory and fieldwork remained integral parts of the curriculum through the end of the Second World War [4]."

It is worth noting that this is one of the few references to "fieldwork" in the engineering education literature. Where it does appear, the term tends to refer to surveying.

The changes in engineering education that flowed from the Grinter report in the mid-1950s meant that laboratory instruction, like the curriculum more generally, took on a more theory rather than practice orientation. The ascendancy of the fundamentals and theoretical knowledge meant that engineering graduates did not acquire the practical understanding of how things worked, something that previously was developed in laboratory classes. Feisel and Rosa [4] observe that this trend towards more theory-centric (academic) courses, combined with the downturn in engineering education in the 1970s, led to laboratory instruction becoming secondary in importance. By the early 1980s, serious concerns were being raised about laboratory instruction in engineering programs. 
"Laboratory oriented instruction for engineering students continues to be a difficult aspect of engineering education. The decreased amount of laboratory instruction in the curriculum, the decreased presence of professorial rank faculty in laboratory instruction, the dwindling level of student interest, and the inadequate resources applied to laboratory oriented instruction are some of the symptoms of the broader, deeper malaise found with this segment of engineering education [5]."

If the laboratory was to regain a central place in engineering curricula, it would be necessary to clearly articulate the purpose and role of laboratory instruction. Ernst [5] posited that laboratories have three major educational objectives, as follows:

1. the student should learn how to be an experimenter,

2. as a place for the student to learn new and developing subject matter and,

3. to help the student to gain insight and understanding of the real world.

Two decades later a more extensive set of Thirteen Objectives were proposed for the engineering laboratory [6]. For convenience, these Objectives are listed in Appendix one of this paper so they can be conveniently referred to by the reader.

This longer list of objectives was created at a time when the conception of a laboratory experience was being challenged by a rise in the use of virtual and remote laboratories. This was driven by a combination of costs associated with maintaining and staffing hands-on laboratories with up-to-date equipment for large numbers of students and the increasing power and sophistication of computer simulations, as well as dramatic expansion in internet bandwidth to support remote labs [4]. While many of the thirteen objectives could apply equally to hands-on, virtual or remote laboratories, a number of them (e.g. Objective 8: Psychomotor and Objective 13: Sensory Awareness) have connotations of a physical experience with hardware while others (e.g. Objective 6: Learn from Failure; and arguably Objective 9: Safety) imply a proximal, and material activity.

The debate about the efficacy of these new types of laboratory experience has continued for nearly two decades, often with the proponents talking at cross purposes. For example, Ma and Nickerson [7] concluded that:

"The debate can be partially explained by examining the educational objectives associated with each laboratory type. Hands-on lab adherents emphasize the acquisition of design skills as an important educational goal, while remote laboratory adherents do not evaluate their own technology with respect to this objective [7]."

The debate about when, why and how to best use hands-on, virtual and/or remote laboratories has also opened up exciting new types of investigations that undergraduate students might be able to undertake. For instance, Lowe, et al [8], highlight how remote laboratory experiences can be created that give access to real-world environments in ways that bounded logistics of a physical laboratory cannot. They use the example of a thermodynamics experiment in a physical lab where students monitor the changing temperature profile of a simple steel block and compare 
this with predications from heat conduction theory. They then contrast the bounded, decontextualized nature of this experience with the more authentic one of an online experiment where the students have access to direct temperature measurements from a casting in a real working foundry located anywhere in the world. Indeed, it is now commonplace for major research agencies including CERN, the EU Space Agency and NASA to make a whole variety of real experimental data available to excite and engage students in schools with contemporary science.

More recently in a review of the relative virtues of physical, virtual and online laboratory experiences in science and engineering education, De Jong et al. [9], conclude that there are circumstances where virtual investigations are equal to or more effective than physical investigations, and times when physical laboratory experiences are the more appropriate. They also note that combinations of the two offer advantages that neither can achieve alone. Thus, we now have a richer palette of experimental experiences with which to develop curricula. Nevertheless, the focus remains primarily on quantitative data about natural phenomena or technical data on the performance of human devised products, processes or systems. Although laboratory experiences (hands-on, virtual or remote) often embed the development of interpersonal knowledge, skills and abilities around communication (Objective 10) and teamwork (Objective 11) and the immediate conduct of the experimentation. The more complex economic, ethical (Objective 12) and cultural aspects of undertaking technical work in the "real world" of practice are absent.

\section{Laboratories \& Accreditation: A Global Perspective}

Since the advent of outcomes-based accreditation of engineering programs in the late 1990s, graduates have been expected to demonstrate certain abilities that are generally assumed have been developed primarily through "laboratory" experiences. The current ABET Criteria 3 (b) [10] requires accredited programs to document that their students graduate with "an ability to design and conduct experiments, as well as to analyze and interpret data". The revised version of this outcome, to take effect in the accreditation cycle 2019-20 and beyond, modifies and expands on the scope of this ability in two important ways. Criteria 3, Outcome 6 states: "an ability to develop and conduct appropriate experimentation, analyze and interpret data, and use engineering judgment to draw conclusions [11]" (Changes are indicated in italics).

Firstly, the change from design to develop implies a more exploratory and emergent process, beyond just being procedural or following a defined set of steps. Combined with the addition of the adjective appropriate this change suggests the need for judgement in conceiving the way in which an engineering investigation is framed, scoped and carried out. Second, analysis and interpretation of data is extended to include the use of engineering judgement in arriving at conclusions and presumably also in the consideration of actions that might follow. The exercise of judgement is a critical professional ability that is usually absent from engineering education criteria.

The addition of engineering judgement echoes the second half of Objective 13: Sensory Awareness in Feisel and Rosa [4] which reads: "Use the human senses to gather information and to make sound engineering judgments in formulating conclusions about real-world problems", (Emphasis added). This is an interesting development given the origins of these Thirteen 
Objectives. They were the outcome of a multi-day colloquy initiated and developed with support by ABET and involving a variety of stakeholder perspectives yet it was made clear at the time that these objectives have "no standing as accreditation criteria [4]". Nevertheless, it seems that concepts put forward in the objectives are now slowly being incorporated into accreditation descriptors.

Around the world, engineering education programs in each national jurisdiction are accredited by the relevant local agency based on the local history and context, e.g. ABET in the US, Engineering Council in the UK, Engineers Canada in Canada, and Engineers Australia in Australia. However, as engineering is a mobile, global profession, and engineers might work in a variety of countries, it became necessary for qualifications gained in one jurisdiction to be recognized in another. In 1989, a foundational group of national accreditation agencies signed the Washington Accord [12] whereby the members agree to recognize engineering qualifications accredited by all the others countries based on the principle of these programs being of "substantial equivalence". The Washington Accord is based upon a generic set of twelve (12) graduate attributes, applicable in all engineering disciplines; what graduates should know, the skills they should demonstrate and the attitudes they should possess. Whereas ABET uses the terms "experiment" or "experimentation" the corresponding attribute in the Washington Accord protocol (WA4) adopts the broader descriptor "investigations" [12]. WA4 is characterized as "Conduct investigations of complex problems using research-based knowledge and research methods including design of experiments, analysis and interpretation of data, and synthesis of information to provide valid conclusions [12]".

This difference is significant as the term "experimentation" can limit our thinking to the bounded notion of a conventional laboratory, whereas "investigation" is more encompassing and thereby open to much wider interpretation of how students might gain these abilities. In addition, the term investigation better captures the nature and variety of the diverse types of work that practicing engineers do that involve gathering and interpreting data in order to draw conclusions about future actions. It also turns out that the design and conduct of experiments and the interpretation of data are not a singular construct. Based on a systemic review of the literature on graduate competencies for undergraduate students, Passow and Passow [13] arrived at several significant conclusions in relation to ABET Criteria 3(b).

"In short, ABET's outcome 3(b) "design and conduct experiments, as well as to analyze and interpret data" is not a valid single competency because "interpret data" rates with top cluster competencies, while "experiments" rates below bottom cluster competencies. Such dramatic differences in the ratings for sub-constructs indicate that combining "experiments" and "interpreting data" hides a valuable distinction in the mind of practicing engineers [13]."

Later in their paper Passow and Passow [13] note that WA 4 also runs counter to combine the conduct of investigations with the interpretation of data and drawing conclusions. These findings have important implications for the design of "laboratory" experiences.

If the development and assessment of abilities around doing "experiments" can be meaningfully decoupled from those associated with "interpreting data", then we can usefully develop learning 
experiences that focus primarily on one or the other. This finding suggests that we need to provide students with more opportunities to focus on analyzing and interpreting datasomething that the research evidence suggests is a distinct competence and indeed much more important than "design and conduct experiments" for engineering practice. Given that engineering practice is not a purely technical undertaking but takes place in a complex and ever changing economic, social, cultural, political and historical context, the data that might need to be analyzed and interpreted could be of many types, technical and non-technical and in many formats, both quantitative and qualitative.

Towards a Richer, Developmental Conception of Laboratory Instruction

In their review of the literature comparing hands-on, simulated and remote laboratories, Ma and Nickerson [7] make the critical observation that in reality, all three types of lab are mediated by the use of computers. Thus, the boundaries between the different types of laboratory were becoming blurred and more nuanced comparisons were required.

"Furthermore, research in psychology suggests that the beliefs and experiences of students may be determined more by the nature of the interfaces than by the objective reality of the laboratory technology. This is a complex issue; it may be that handson labs are important initially to establish the reality of remote laboratories or the accuracy of simulations for later study.

Finally, it is clear that students learn not only from equipment, but from interactions with peers and teachers [7]."

This observation highlights the developmental aspect of becoming an effective investigator. It also recognizes the social dimension of learning.

There is a whole body of pedagogical literature that recognizes this developmental dimension and the need for appropriate scaffolding and support from peers and teachers. Laboratory experiences can be characterized in terms of the degree of autonomy afforded to the students; the degree to which they can exercise agency in what is investigated, how this is done and what conclusions are drawn. For instance, building on earlier work by Herron [14] and others, Buck et al. [15] developed a rubric of types of undergraduate laboratory experience as shown in Table 1. Similar rubrics have been developed by others (e.g. [16]) and such rubrics have been accepted into best practice summaries for laboratories (e.g. [17], [18] ).

\begin{tabular}{|c|c|c|c|c|c|}
\hline Characteristic & $\begin{array}{c}\text { Level 0: } \\
\text { Confirmation }\end{array}$ & $\begin{array}{c}\text { Level 1/2: } \\
\text { Structured Inquiry }\end{array}$ & $\begin{array}{c}\text { Level 1: } \\
\text { Guided Inquiry }\end{array}$ & $\begin{array}{c}\text { Level 2: } \\
\text { Open Inquiry }\end{array}$ & $\begin{array}{c}\text { Level 3: } \\
\text { Authentic Inquiry }\end{array}$ \\
\hline Problem/Question & \multirow{6}{*}{ PROVIDED } & \multirow{4}{*}{ PROVIDED } & \multirow{3}{*}{ PROVIDED } & \multirow{2}{*}{ PROVIDED } & \multirow{6}{*}{$\begin{array}{c}\text { NOT } \\
\text { PROVIDED }\end{array}$} \\
\hline Theory/Background & & & & & \\
\hline Procedures/Design & & & & & \\
\hline Results Analysis & & & & $\begin{array}{c}\text { NOT } \\
\text { PROVIDED }\end{array}$ & \\
\hline Results & & & $\begin{array}{c}\text { NOT } \\
\text { PROVIDED }\end{array}$ & & \\
\hline Conclusions & & PROVIDED & & & \\
\hline
\end{tabular}

Table 1 Characteristics of Different Levels of Inquiry in UG Laboratory [15] 
There is a learning progression from level 0 to level 3 as students develop their knowledge, skills, abilities and confidence to undertake more open-ended inquiry. With each step in this progression, the student is given increasing amounts of autonomy in making decisions about how to conduct the investigation, and eventually what to investigate and why. This aligns with the developmental assumption underlying the Thirteen Objectives; these were always intended to be cumulative and apply over the entire undergraduate program and not be the outcome of any one laboratory experience [6]. With the possible exception of a capstone research project, arguably few undergraduate students get to experience a level 3 lab.

Several of the Thirteen Fundamental Objectives of Engineering Instructional Laboratories [4] point to a broader conception of what might be encompassed by lab. For instance, the inclusion of Objective 5: Design and Objective 7: Creativity reflect the inductive and generative thinking that is an integral part of engineering investigations and "real-world" problem solving. Viewed another way, the inclusion of these two objectives reminds us that design and creativity both involve investigatory elements, exploration, data and information gathering, analysis and interpretation, often through the design and conduct of experiments. The power of design thinking by Brown [19] with its emphasis on early and frequent prototyping to test ideas, physically or virtually, is a manifestation of the interdependence between engineering design and engineering investigation. The contemporary mantra associated with design thinking and innovation, "fail early and fail often" overlaps with the title of Objective 6: Learn from Failure. While not mentioned explicitly, this objective implies that we should be developing students' ability to conduct disciplined and critical reflections about the broader lessons learned as they engage in engineering experimentation or other investigations.

The first part of Objective 13: Sensory Awareness refers to the "use the human senses to gather information". Taken narrowly, this might refer to using senses to understand physical or chemical phenomena; or otherwise explore the behavior of the material world. More broadly, it can include nuanced ethnographic observation of how people interact with, use and behave around engineered products or systems or relate to new technologies. Thus, the sophisticated use of all the senses is even more powerful when conducting a technical investigation in the context of an unfamiliar socio-cultural environment, as occurs in global engineering fieldwork. Moving from the straightforward use of a few of our senses to being able to accurately observe, faithfully record and meaningfully interpret human interactions in a technological setting implies a sophisticated developmental progression.

Similarly, Objective 12: Ethics in the Lab, takes on additional layers of complexity once the scope of the work and the modes of gathering, sharing and interpreting data are established within the wider social context in which it is conducted. The technical is embedded in the cultural. Choices around what is investigated, and when and how this is done, are bound up in cultural values and the prevailing practices. Determining what practices should prevail in a lab setting can become embroiled and confounded by matters of societal equity and emergent technology. Considerations of power distance [20] shed light on the fact that individuals in a lab setting may operate and be treated unequally based on dimensions ranging from apparent intellect to attributes associated with national culture. Anticipating these "hidden in plain sight" realities bound in the lab setting can expose the many ethical considerations students may 
someday encounter. Likewise, the emergence of large data sets (i.e. big data), and the availability of all types data collected and posted on the world-wide web raise new operational questions. Engineers' use of this information raise critical ethical questions regarding what is being analyzed, access to and timing of analysis, and even how and to whom the results and inferences are communicated [21]. Thus, the ethical dilemmas embedded into practices associated with traditional laboratories demand greater socio-cultural awareness and sensitivity.

It is proposed that technical laboratory classes, typically at levels 0 to 2 (Table 1 ) be augmented by an open engineering investigation in a socio-cultural context similar to that experienced by practitioners undertaking engineering fieldwork. Developmentally, this gives the students an opportunity to translate their knowledge, skills and abilities gained in "technical" labs into a more realistic operating environment. While the problem and maybe some of the background would be provided (as in a level 2 technical lab), the learning challenge is more akin to that of a level 3 laboratory experience, particularly in problem scoping, which necessarily leads to the realm of creating specifications, implementing procedures, and interpreting results.

In the practice of engineering, in particular fieldwork, the data collection, interpretation, and problem solving domain is rarely restricted to situations purely focused on the technical solution. To that end, spending ample time investigating and uncovering elements of the broad problem space often differentiates expert engineering behaviors from those of beginners [22]. Likewise, as the engineer begins to uncover the essence of the fieldwork at hand, it is likely that a range of data types may come from a variety of stakeholders, areas of expertise, depth of skill (or possible lack thereof) and so on. Having available engineering experts at hand to scaffold student's processing through the foreign new and overwhelming, aids in building their patience and capability to engage variety.

Upon examination, the apparently straight forward lab tasks of identifying design/test criteria, documenting experiments, and interpreting data sets, suddenly requires acquisition and exercise of professional habits. These tasks may include developing the ability to provide clear and frequent communication across cultural normative practices, negotiate technical and nontechnical vocabulary understanding, level-set base operating assumptions, and even determine how best to convey engineering meaning through the use of diagrams, sketches, or physical models.

Developing a Socio-Technical Lab Experience based around a Global Field Investigation

To test this broader conception of a laboratory experience encompassing socio-technical investigations, we designed an intensive, one-week study abroad course to emulate the experience of a practicing engineer; one who is required to travel to a remote, unfamiliar global location to conduct engineering fieldwork and return home in a week [1]. Field investigations, especially those conducted in a global context, involve many different types of data, not only engineering (technical) data but also relevant economic, social, cultural, geographic, historic and other data. This highly integrated set of technical and cultural experiences provided an authentic context in which the students could develop observational, analytical and interpretive skills that went beyond that feasible in a conventional laboratory setting. 


\section{Learning Goals}

The overarching goal of this accelerated, seven day field course was to produce authentic engineering experiences and artefacts integrated with the development of cultural awareness.

Through participation in it, the goal was for students to develop their ability to:

- Plan \& execute an open-ended engineering investigation in an unfamiliar cultural setting.

- Identify, gather, log, analyze, and interpret field data using multiple approaches.

- Present ideas orally and visually in less formal modes during the course of an investigation.

- Undertake a structured reflection in order to learn lessons from the experience.

With respect to planning and conducting a field investigation, the enduring understandings that the students were expected to acquire were:

- Disciplined approaches to making observations, unearthing stakeholder issues, making effective field notes and integrating information from diverse sources are essential skills for engineers.

- Success conducting an investigation depends critically on understanding the relevant cultural context.

- Effective communication (including listening) must be attuned to disciplinary differences and the cultural context of the end user in order to identify the real problem to needs to be investigated.

A series of knowledge and skills were conceived and carefully mapped to these course goals and enduring understanding. As a result of the study abroad experience the students were expected to know the following:

- The critical need to first clarify the task and set measurable objectives when planning experiments.

- The impact that careful planning has on the successful execution of an engineering investigation.

- Sources of possible error in field data and how to interpret incomplete data from disparate sources.

- That cultural style differences exist and why these matter when working in global teams.

- Effective communication requires awareness of different cultural values, norms, and practices.

- How to orally present experimental findings in a concise and culturally aware fashion.

The skills they were expected to be able to acquire were as follows:

- Use PM and Agile practices to plan an engineering investigation of an ill-defined problem.

- Use keen observation and other methods to collect data in a "messy", real world environment.

- Use multiple techniques \& technologies to record data in an engineering field investigation. 
- Take neat, clear, concise, accurate and useful field notes in an effective and efficient manner.

- Test a working hypothesis through experiments involving iterative data collection and analysis.

- Make reasonable estimates of inaccessible data / missing information sufficient for the investigation.

- Integrate diverse data types from many sources as part of conducting an experimental investigation.

- Make effective, impromptu presentations of findings to a diverse audience of stakeholders.

- Conduct a disciplined reflection to improve knowledge and skills of how to conduct experiments.

The course was designed principally for students in a Multidisciplinary Engineering Program. This ABET accredited program attracts students who have strong academic interests beyond just engineering and who undertake one of a wide variety of plans of study that include courses selected from across the engineering disciplines as well as from the Liberal Arts or the Sciences.

\section{Student Preparation}

Significant intention was placed on helping the students situate the cultural learning components within all aspects of the trip. Three pre-trip "orientation" sessions challenged students to develop their observation and reflection skills, by exercising tools to support their nascent cultural understanding, and bring forward that awareness to inform their future engineering solutions. This preparation included becoming familiar with the use of cultural self-awareness [23] to identify and explore cultural differences, mindfulness activities as a means to bring forth situational clarity, and manage times of stress [24], and incorporation of a structured approach to harvest reflections around critical incidents for data collection. Exposure to these practices was established to form a foundation of common field practices, as well as a language to draw upon while abroad. The critical incident reflection technique SAID-Situation-Affect-InterpretationDecision [25] was used to help unpack and document individual experiences, to share them with their cohort, and facilitate discussion on confusing cultural cues relevant to their projects.

\section{A Global Engineering Investigation}

Simulating a demanding field investigation in a remote context is authentic in that many practicing engineers will share like experiences at some point in their career. Creating authenticity in the global engineering investigation during a short-term study abroad course, is easier said than done. Short-term study abroad coursework with such an aim is challenging because to meaningfully incorporate both technical and local cultural elements requires making strong connection between the two, else the students compartmentalize the experiences, failing to make gains associated with integration of both. The choice of destination location and selection of the experiential focal point must work synergistically to both scaffold the unfamiliar and uncertain, while concurrently providing ample room for exploration, meta-examination, informed trial and error, and sufficient risk taking. 
This example study abroad course experience involved eight U.S. students flying to Australia for a week to join a Global Design class already underway at Swinburne University of Technology in Melbourne. This destination was chosen so that the visiting students were not overwhelmed by major cultural and linguistic barriers (as would be the case travelling to say, East Asia). This apparent familiarity enabled them to quickly assimilate, join in, and work seemingly effectively with the local team. Each U.S. student worked with a pre-existing local team of three Product Design Engineering (PDE) students who were three weeks into a semester long project to design an innovative product likely to mitigate the negative effects of annual flooding found in the urban setting of Kampung Melayu in Jakarta, Indonesia. This challenge arose from an ongoing research collaboration between Swinburne and a university in Indonesia and so the students had access to an extensive range of contextual information (including photographs and maps) and access to staff familiar with the Indonesian cultural context.

Day one on the visiting campus for the U.S. students consisted of a two-hour briefing by the local students on the project as they understood it, plus their initial solution ideas. The U.S. students were then challenged to undertake an intensive exploration of the project and report back at the next class session to the local students, on day four of their visit. The students embarked upon intensive socio-cultural and technical data collection and began early analysis. This data was generated through a semi-structured briefing session with a post-doctoral scholar from Indonesia, engaged in research on humanitarian design. The students were able to question the expert about the context of Kampung Melayu, as well as independently seeking out sources of technical and non-technical information from local engineering experts and the World Wide Web.

Over the subsequent three days through an iterative cycle of examining, filtering, analyzing, and interpreting data and then operationalizing the information via rapid prototyping of design concepts, the students gained deeper and deeper insights around the nature and scope of the problem. This process was punctuated by periodic impromptu concept presentations, plus group and individual reflections on stakeholder needs, design criteria, etc. When the students reported back to the PDE class, it consisted of a poster presentation with supporting evidence and demonstration of a physical artefact. Through a rigorous Q\&A session the visiting students communicated and defended their design choices. Since the local students had not met as a class since day one, the expectation was that the visitor presentations would help the local students progress their own designs, and perhaps rethink their impressions of important contextual issues.

The intensive investigation activities around the Jakarta project was interspersed with short technical visits and workshops, one including rapid idea generation, development and testing using low-fidelity prototyping; others included visiting the state of the art Advanced Manufacturing and Design Centre (AMDC). These technical excursions were complemented by cultural experiences, some during the day and some in the evening. A reason for choosing Melbourne, is the large, vibrant and proudly multi-cultural nature of the city, with immigrants drawn from over 180 countries. This location provided a rich tapestry of cultural diversity for the U.S. students to be immersed in, during structured work time, or during their so-called free time. 
Learning to Make Engineering Judgements in the Field

Task oriented accomplishments associated with a reality-based laboratory experience for students abroad are not difficult to count and measure, however capturing the learning gains can be elusive. This is in part due to the deconstruction of formalized learning settings and traditional rigidly prepared assessment activities that follow, in favor of more investigative and emergent encounters and unscripted breakthroughs found in live field action.

Difficulty in measurement aside, archetypal incidents of learning emerged within the program that had a material effect on the student's designs and work product. Under closer examination, the incidents logged by the instructors were bound by a common theme of what became referred to as "realizations beyond technical" concern, or RBT for short. Two examples of such contextual realizations materialized in student designs related to the Indonesian culture specifically in regard to family roles/order, and taboos. Through qualitative data collection, it was noted by students that family roles and associated practices within the dominant culture dictated how the population responded in times of flooding crisis. Students articulated in presentations that $\mathrm{men} / \mathrm{husbands}$ were expected to stay behind to stand guard and protect their family wealth and earthly possessions, while women/wives were tasked with evacuation of children, and elderly family members. These realizations lead to ideation, design criteria, and solutions around securing family items and enabling safer evacuation for women. Investigation of prior art, research of existing tools/products used by the community, and rapid ideation sessions followed. Students began formulating detailed product designs and prototypes such as: built in lock boxes located in accessible but hidden second story floor joists; collapsible, second story window mounted lockers; pontoon animal carriers that float in high water and serve as everyday shelter; and a multipurpose wheelbarrow-esque shelter and transport unit. It should be noted, that many of these ideas were completely new, once the insights around family roles registered with the students.

The need to pivot with their design concepts was also experienced when students faced unexpected roadblocks around user acceptance related to bodily function taboos. Several students were forced to set aside their "ideal" designs when they discovered that approaches to addressing disease prevention from unprocessed sewage in flood water was more difficult than simply designing a eco-friendly port-o-potty for the impacted community to share. Religious influences among the population not only deemed their proposed solution sketches as ineffectual, but also offensive and in direct violation of the norms of the community. Ultimately, the impacted U.S. students retained their design focus on clean water, but settled on designing a unit to purify and harvest rainwater for drinking; lowering the risk related to possible concerns and criteria they now felt far less capable of designing a solution for.

Vygotskian theory [26] would suggest that student learning most likely occurred as they engaged in the socio-cultural examination of their designs through feedback of the target user group.

Recent research on quantity and quality of student learning suggest that incidents of cultural dissonance create both the "means and medium of intercultural learning" [27]. According to the research and using this example, students who successfully navigated through such an experience will likely improve cognition, empathy, reflection and self-confidence. 


\section{Some Lessons Learned}

As this laboratory course was both new and different in its approach, we were keen to document what worked and what might need improvement going forward. In consideration of level four laboratory experiences, lessons revolve around the themes of destination location, critical pedagogical review, student reflection, and overall course assessment approaches.

\section{Selecting the Location}

At first glance a study abroad trip to Australia might not seem to present any major cultural challenges for U.S. students, however the apparent similarities between the two countries can be used to advantage. Socio-cultural settings that are superficially familiar sometimes turn out to be quite different in subtle ways the closer you get to them. Being able to immediately communicate and work effectively with the local students and instructors, meant that the visitors could readily observe and pick up on the ways that things were not the same as at home. Not being overwhelmed by unusual sights and sounds and unfamiliar practices of a "foreign" country enabled the student visitors to self-reflect and gain insights about their home culture and make visible some implicit operating assumptions about what is 'normal' to them for global engineering fieldwork and practice.

The Product Design Engineering program is a structured blend of courses drawn from engineering and industrial design, so this provided the visiting students with peer collaborators who, like them, had a broad understanding of what engineering is and can be. Yet the skill sets of the two groups were distinct. For instance, the PDE students had advanced ability to sketch and represent ideas visually, which encouraged the visiting students to raise their game, to compete in the quality of visual presentations. Like the selection of the location, the choice of the students that the visitors were going to interact with was very intentional; instant familiarity yet underlying difference that might lead to dissonance and discovery.

\section{Reflection}

Reflective practices utilized throughout the laboratory were prescribed to develop student skills around navigating problem context, resolving aspects of data collection and sense making, and finalizing design proposals/decision making. While presented to students as an essential habit of professional engineering practice, it was observed that even when provided with a structural methodology for documenting their experiences, students were unable to go deep to elaborate upon their observations, inferences, and pivot points of decision making throughout the project. Rather than understanding the methodology as a means for elucidating their thoughts and feelings, they viewed the method as a repetitive exercise, which often resulted in them providing redundant responses. This highlights the need in engineering education for greater explanation of how and why engineering professionals find value in engaging in such reflective exercises. In

this instance of the course, it suggests that more face to face scaffolding time may be required to help students develop their skills and abilities in this area. Providing such intervention in the future may help students see potential long-term professional benefits of mastering the method.

\section{Assessment}

In the domain of educational assessment, there is a balance to strike between what we seek to measure (the valued outcomes) versus what is easily measured (available outcomes). Fieldwork which operates within a space of some uncertainty presents an assessment challenge. It must 
highlight value in available assessments that are easily planned and collected, while concurrently innovating ways to capture the illusive but highly valued learning outcomes that perhaps occur in unscripted fashion.

Based on this first execution of the study abroad course, capturing and aligning incidents of assessment to learning outcomes is a task that requires dedicated and detailed attention to achieve robust results. While both time and attention was put forth in the preplanning and alignment of course objectives, learning outcomes, and assessment approaches, gaps existed when reviewing the course from a continuous improvement perspective. Future iterations of the course (or like courses) will benefit from additional reflective examination in this area, in order to document and claim learning gains associated with the program.

\section{Conclusion}

Laboratory experiences of engineering students have morphed and expanded significantly over the past century. While conventional laboratory instruction was once focused on hands-on activities, virtual and computer-based simulation and remote labs are now commonly accepted forms of lab practice. Expanded language used around experimentation in the new ABET Criteria 3 should encourage engineering educators to innovate around what it means to engage engineering investigations including the laboratory, beyond the purely technical domain.

This paper presents a review of the literature and qualitative support demonstrating an attempt toward this expanded view of experimentation and laboratory. Through the lens of a case-study, "socio-technical" elements and experiences in the ubiquitous form of engineering experimentation vis-à-vis fieldwork, are elaborated upon. The novel study abroad course described provides an instance of how to emulate practices natural to global engineering fieldwork and develop essential technical and professional skills such as problem identification, decision making and reflection. Future work in this area is needed in order to improve the collection and articulation of student learning gains associated with authentic engineering fieldwork.

\section{Acknowledgements}

We wish to thank Dr. Charles Ranscombe, and Dr Yenny Rahmayati and the PDE students plus Dr. Emily Wright and the Design Factory Melbourne for welcoming us into their classes. We are indebted to Dr. Robin Adams, Ms. Dorothy Missingham, Mr. Geza Lanczy, and training personnel of Purdue CILMAR for sharing technical and cultural expertise, and generously offering their time for informal student debriefs and cultural scaffolding along the way.

\section{References}

[1] M. K. Pilotte and D. F. Radcliffe, "Preparing students for intensive global fieldwork," in 2017 IEEE Frontiers in Education Conference (FIE), 2017, pp. 1-5: IEEE.

[2] R. M. Reck, "Applying a common framework to develop undergraduate control systems laboratory kits," in 2017 IEEE Frontiers in Education Conference (FIE), 2017, pp. 1-8. 
[3] C. R. Mann, "A study of engineering education: prepared for the Joint Committee on Engineering Education of the National Engineering Societies," in "The Carnegie Foundation for the Advancement of Teaching", Boston, 1918.

[4] L. D. Feisel and A. J. Rosa, "The role of the laboratory in undergraduate engineering education," Journal of Engineering Education, vol. 94, no. 1, pp. 121-130, 2005.

[5] E. W. Ernst, "A new role for the undergraduate engineering laboratory," IEEE Transactions on Education, vol. 26, no. 2, pp. 49-51, 1983.

[6] L. D. Feisel, G. D. Peterson, O. Arnas, L. Carter, A. Rosa, and W. Worek, "Learning objectives for engineering education laboratories," in Frontiers in Education, 2002. FIE 2002. 32nd Annual, 2002, vol. 2, p. F1D: IEEE.

[7] J. Ma and J. V. Nickerson, "Hands-on, simulated, and remote laboratories: A comparative literature review," ACM Computing Surveys (CSUR), vol. 38, no. 3, p. 7, 2006.

[8] D. Lowe, S. Murray, E. Lindsay, and D. Liu, "Evolving remote laboratory architectures to leverage emerging internet technologies," IEEE Transactions on learning technologies, vol. 2, no. 4, pp. 289-294, 2009.

[9] T. De Jong, M. C. Linn, and Z. C. Zacharia, "Physical and virtual laboratories in science and engineering education," Science, vol. 340, no. 6130, pp. 305-308, 2013.

[10] Engineering Accreditation Commission of the Accreditation Board for Engineering and Technology. (2014, April 17). Accreditation Policy and Procedures Manual. Available: www.abet.org

[11] Engineering Accreditation Commission of the Accreditation Board for Engineering and Technology. (2018, January 22). Criteria for Accreditation Engineering Programs. Available: http://www.abet.org/accreditation/accreditation-criteria/criteria-foraccrediting-engineering-programs-2018-2019/

[12] IEA. (2014). Washington Accord, International Engineering Alliance Secretariat. Available: http://www.ieagreements.org/assets/Uploads/Documents/History/25YearsWashingtonAc cord-A5booklet-FINAL.pdf

[13] H. J. Passow and C. H. Passow, "What Competencies Should Undergraduate Engineering Programs Emphasize? A Systematic Review," Journal of Engineering Education, vol. 106, no. 3, pp. 475-526, 2017.

[14] M. D. Herron, "The nature of scientific enquiry," The School Review, vol. 79, no. 2, pp. 171-212, 1971.

[15] L. B. Buck, S. L. Bretz, and M. H. Towns, "Characterizing the level of inquiry in the undergraduate laboratory," Journal of College Science Teaching, vol. 38, no. 1, p. 52, 2008.

[16] E. Hazel and C. Baillie, "Improving teaching and learning in laboratories," Higher Education Research \& Development Society of Australasian, no. 4, 1998.

[17] C. Davies, Learning and teaching in laboratories. (C) Higher Education Academy Engineering Subject Centre, Loughborough University, 2008.

[18] L. Kirkup and L. Johnson, "Threshold Learning Outcome 3-Inquiry and Problemsolving," Office for Learning and Teaching Good Practice Guide (Science), 2013.

[19] T. Brown, "Change by design," 2009.

[20] G. Hofstede, G. J. Hofstede, and M. Minkov, Cultures and Organizations: Software of the Mind, 3rd ed. Columbus: McGraw-Hill, 2010. 
[21] D. Boyd and K. Crawford, "Critical questions for big data: Provocations for a cultural, technological, and scholarly phenomenon," Information, communication \& society, vol. 15, no. 5, pp. 662-679, 2012.

[22] C. J. Atman, R. S. Adams, M. E. Cardella, J. Turns, S. Mosborg, and J. Saleem, "Engineering design processes: A comparison of students and expert practitioners," Journal of engineering education, vol. 96, no. 4, pp. 359-379, 2007.

[23] C. A. Calahan. (2017). Knowledge of Cultural Self-Awareness Assignment. Available: https://www.purdue.edu/cie/Documents/Global\%20Learning/c self aware.pdf

[24] J. Dohaney, E. Brogt, and B. Kennedy, "Strategies and Perceptions of Students' Field Note-Taking Skills: Insights From a Geothermal Field Lesson," Journal of Geoscience Education, vol. 63, no. 3, pp. 233-249, 2015.

[25] C. Hogan, "Creative and reflective journal processes," The Learning Organization, vol. 2, no. 2, pp. 4-17, 1995.

[26] L. S. Vygotsky, Mind in Society. Cambridge, MA: Harvard University Press, 1978.

[27] M. Allan, "Frontier crossings: Cultural dissonance, intercultural learning and the multicultural personality," Journal of Research in International Education, vol. 2, no. 1, pp. 83-110, 2003. 


\section{Appendix 1: Objectives of Engineering Laboratories (Fiesel and Rosa, 2005) [4]}

All objectives start with the following: "By completing the laboratories in the engineering undergraduate curriculum, you will be able to...."

\begin{tabular}{|c|c|}
\hline $\begin{array}{l}\text { Objective 1: } \\
\text { Instrumentation. }\end{array}$ & $\begin{array}{l}\text { Apply appropriate sensors, instrumentation, and/or software tools to make measurements } \\
\text { of physical quantities. }\end{array}$ \\
\hline Objective 2: Models. & $\begin{array}{l}\text { Identify the strengths and limitations of theoretical models as predictors of real-world } \\
\text { behaviors. This may include evaluating whether a theory adequately describes a physical } \\
\text { event and establishing or validating a relationship between measured data and underlying } \\
\text { physical principles. }\end{array}$ \\
\hline $\begin{array}{l}\text { Objective 3: } \\
\text { Experiment. }\end{array}$ & $\begin{array}{l}\text { Devise an experimental approach, specify appropriate equipment and procedures, } \\
\text { implement these procedures, and interpret the resulting data to characterize an } \\
\text { engineering material, component, or system. }\end{array}$ \\
\hline $\begin{array}{l}\text { Objective 4: Data } \\
\text { Analysis. }\end{array}$ & $\begin{array}{l}\text { Demonstrate the ability to collect, analyze, and interpret data, and to form and support } \\
\text { conclusions. Make order of magnitude judgments and use measurement unit systems and } \\
\text { conversions. }\end{array}$ \\
\hline Objective 5: Design. & $\begin{array}{l}\text { Design, build, or assemble a part, product, or system, including using specific } \\
\text { methodologies, equipment, or materials; meeting client requirements; developing system } \\
\text { specifications from requirements; and testing and debugging a prototype, system, or } \\
\text { process using appropriate tools to satisfy requirements. }\end{array}$ \\
\hline $\begin{array}{l}\text { Objective 6: Learn } \\
\text { from Failure. }\end{array}$ & $\begin{array}{l}\text { Identify unsuccessful outcomes due to faulty equipment, parts, code, construction, } \\
\text { process, or design, and then re-engineer effective solutions. }\end{array}$ \\
\hline $\begin{array}{l}\text { Objective 7: } \\
\text { Creativity. }\end{array}$ & $\begin{array}{l}\text { Demonstrate appropriate levels of independent thought, creativity, and capability in real- } \\
\text { world problem solving. }\end{array}$ \\
\hline $\begin{array}{l}\text { Objective 8: } \\
\text { Psychomotor. }\end{array}$ & $\begin{array}{l}\text { Demonstrate competence in selection, modification, and operation of appropriate } \\
\text { engineering tools and resources. }\end{array}$ \\
\hline Objective 9: Safety. & $\begin{array}{l}\text { Identify health, safety, and environmental issues related to technological processes and } \\
\text { activities, and deal with them responsibly. }\end{array}$ \\
\hline $\begin{array}{l}\text { Objective 10: } \\
\text { Communication. }\end{array}$ & $\begin{array}{l}\text { Communicate effectively about laboratory work with a specific audience, both orally and } \\
\text { in writing, at levels ranging from executive summaries to comprehensive technical } \\
\text { reports. }\end{array}$ \\
\hline $\begin{array}{l}\text { Objective 11: } \\
\text { Teamwork. }\end{array}$ & $\begin{array}{l}\text { Work effectively in teams, including structure individual and joint accountability; assign } \\
\text { roles, responsibilities, and tasks; monitor progress; meet deadlines; and integrate } \\
\text { individual contributions into a final deliverable. }\end{array}$ \\
\hline $\begin{array}{l}\text { Objective 12: Ethics } \\
\text { in the Laboratory. }\end{array}$ & $\begin{array}{l}\text { Behave with highest ethical standards, including reporting information objectively and } \\
\text { interacting with integrity. }\end{array}$ \\
\hline $\begin{array}{l}\text { Objective 13: } \\
\text { Sensory Awareness. }\end{array}$ & $\begin{array}{l}\text { Use the human senses to gather information and to make sound engineering judgments in } \\
\text { formulating conclusions about real-world problems. }\end{array}$ \\
\hline
\end{tabular}

